\title{
Experimental Results on Multicarrier MIMO HF Communications
}

\author{
Ivan Perez-Alvarez ${ }^{1}$, Santiago Zazo-Bello ${ }^{2}$,Mounir Ghogho ${ }^{3,4}$, Javier Lopez-Perez ${ }^{1}$ \\ ${ }^{1}$ Instituto para el Desarrollo Tecnológico y la Innovación en Comunicaciones (IDeTIC), Dept. Señales y \\ Comunicaciones, Universidad de Las Palmas de Gran Canaria. Parque Científico-Tecnológico, Edif. Polivalente II, \\ 35017, Las Palmas de G.C., Spain. tel: +34 928457362, fax:+34 928400040, iperez@dsc.ulpgc.es \\ ${ }^{2}$ ETSI Telecomunicación, Dept. SSR, Universidad Politécnica de Madrid. Avenida Complutense no 3028040 , \\ Madrid, Spain. tel: +34 915495700(4042), fax:+34 913367350, santiago@gaps.ssr.upm.es \\ ${ }^{3}$ School of Electronic and Electrical Engineering, University of Leeds. LS2 9JT, Leeds, UK. \\ tel: +44 1133432054, fax: +44 1133432032, m.ghogho@ieee.org \\ ${ }^{4}$ International University of Rabat, Morocco
}

\begin{abstract}
Achieving reliable communication over HF channels is known to be challenging due to the particularly hostile propagation medium. To address this problem, diversity techniques were shown to be promising. In this paper, we demonstrate through experimental results the benefits of different diversity strategies when applied to multi-inputmulti-output (MIMO) multicarrier systems. The performance gains of polarisation, space and frequency diversities are quantified using different measurement campaigns.
\end{abstract}

\section{Introduction}

Communication in High Frequency (HF) band is not considered to be reliable, due to large variations and highly dispersive characteristics of the HF channel: frequency selectivity due to multipath, Doppler spread, noise and interferences. Multicarrier modulation, specifically Orthogonal Frequency Division Multiplex (OFDM), is an appropriate approach for combating frequency-selectivity of the channel and narrowband interference. The idea is quite simple, yet very effective to avoid complex receivers and to provide more reliable communication. By sending a parallel set of narrowband signals, the channel can be approximated as frequency flat over each signal's bandwidth. This modulation leads to simple frequency-domain channel equalisation, consisting of one complex coefficient per subcarrier. Although frequency selective channels are well dealt with using OFDM, HF channel still remains hostile due to random signal attenuation affecting the whole band, and the presence of multiple sources of interferences due to human activity and atmospheric phenomena. The way to mitigate such impairment is to exploit diversity, which enables to transmit over desirably uncorrelated channels.

This paper describes parts of the work developed in the M3HF project (Multicarrier, Multiband, Multiantenna systems in HF communications, www.m3hf.eu), whose main goal is to propose practical solutions to increase reliability and capacity in HF communications. Theoretical and simulated studies are corroborated by real links for different diversity strategies: i) frequency bands to exploit the incoherence amongst them, ii) multicarrier exploiting two dimensional (time and frequency) coding and interleaving, and iii) antenna diversity through radiation pattern, and spatial and polarisation diversities. The results shown here focus on the last two strategies, multicarrier and antenna diversities; for the latter, we focus particularly on spatial and polarization diversities.

A link in HF communications is typically established between a fixed ground station and a mobile station: vehicle, ship or airplane. Ground stations usually do not have operational restrictions, unlike mobile stations which are subject to stringent restrictions on power, space or antennas. These restrictions severely limit the possible diversity strategies that may be implemented at transmission. Even for ground stations, space-time or spacefrequency diversity implementations using Alamouti codes [1,2] are extremely complex due to antennas coupling (transmission power can be several hundreds of Watts). Large separation between antennas avoids such a coupling, but introduces the critical issue of synchronization amongst transmitters. At present, we do not have a working implementation of multi-antenna transmission; the transmit diversities implemented in our system are: time, frequency, multicarrier and multiband. At reception, frequency and polarisation diversities seem to be the most suitable as space is a critical restriction. Moreover, polarisation diversity is the only strategy that keeps the same 
system parameters (power, bandwidth, throughput, etc.) as in single-input-single output (SISO) system, which is an important aspect if we are to compare performance of the new and legacy systems.

For a long time the scientific community has been experimenting with diversity in HF communications. A comparison of space and polarisation diversity is made in [3] concluding that both systems are equivalent. In [4] frequency and polarisation diversities are compared, and the latter can provide significantly better results than the former. In [5] polarisation diversity was shown to significantly improve bit error rate (BER) and link reliability. However, to the best of our knowledge, no studies on HF have been carried out using multicarrier transmissions with the combination strategies proposed in this paper. The combination strategies proposed in the previous studies merely consisted of signal selection or simple addition of signals corresponding to different diversity branches.

\section{System Description}

The HF stations are located in Gran Canaria (Canary Islands) and Madrid, Spain: a HF mid-latitude 1800km long link from south to north. The receiving station has two locations: Madrid city with a Yagi antenna (location A), which is the same antenna used at the transmitting station, and El Casar with two dual monopole vertical-horizontal polarised antennas (location B). Locations $\mathrm{A}$ and $\mathrm{B}$ are $40 \mathrm{Km}$ apart, and the distance between the two antennas at location B is 75 meters. Figure 1 (b) shows the block diagram of the signal path in location B, where the received signal feeds four R\&S EK895 receivers and the audio signal is sampled and fed to the HFDVL (HF Data+Voice Link) modem, which is described below. All receivers are configured similarly and no actions are taken on the

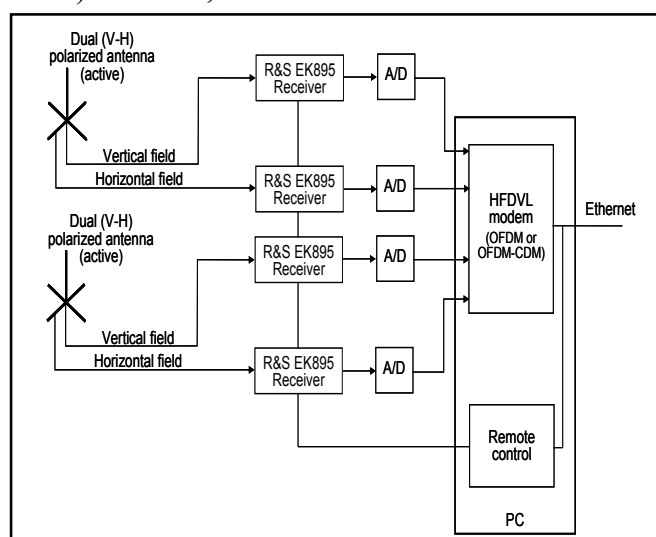

Figure 1: Block signal path in location B. received signal prior to being input into the modem. The transmitter site works with a $1 \mathrm{Kw}$ amplifier, but the RMS power measured at the amplifier's output is lower than $300 \mathrm{w}$ due to the multicarrier signal's high Peak to Average Power Ratio (PAPR).

The HFDVL modem is basically a plain OFDM modem with the same structure for digital voice and data communications. The former is described in detail in [6] and uses a very efficient delay-sensitive MC-CDMA signalling (OFDM-CDM). The modem has the following characteristics: signal bandwidth is $2.7 \mathrm{KHz}$ with 73 carriers (60 for data and 13 interleaved pilots), subcarrier spacing is $37.5 \mathrm{~Hz}, 4 \mathrm{QAM}$ constellation, 30 OFDM symbols per second, and cyclic prefix of more than 3 milliseconds. There are important enhancements in the data modem necessary to deal with the requirement of data transmissions. Indeed, voice requires a lower error rate (BER around $10^{-2}$ when using a MELP vocoder is sufficient). Also, data is typically not limited by severe delay constraints. Therefore, for data transmission we adopt a robust coding strategy: LDPC codes using LLR as soft bits with code rates of $1 / 2$ or $4 / 5$. Besides, in order to provide a wide range of performance results, higher size constellations were implemented (up to 64QAM). Figure 2 shows a block diagram with the main aspects of the modem. A detailed description and performance can be found in [7].

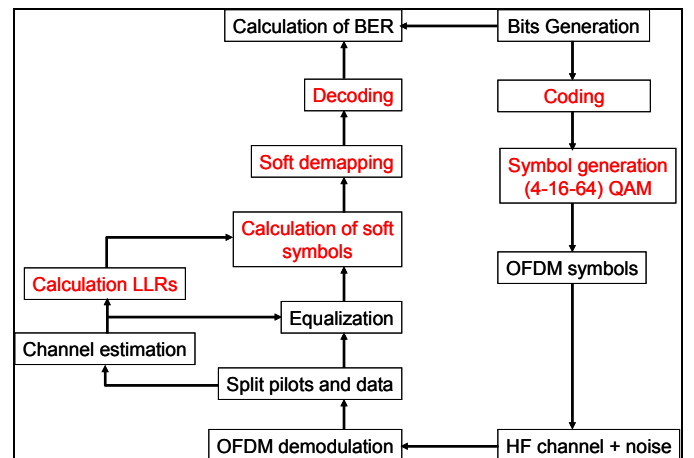

(a)

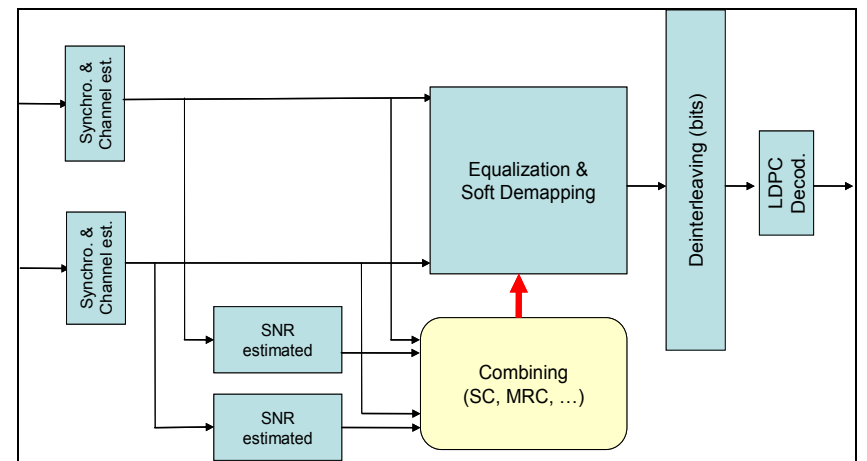

(b)

Figure 2: (a) SISO block diagram of data modem (black blocks are common with digital voice modem); (b) block diagram of reception part of HFDVL when multiple inputs are present. 
When multiple inputs are present, all received signals are demodulated in parallel and then combined in order to recover the transmitted OFDM symbol. Although several combination techniques have been implemented, we will focus here on: OFDM symbol Selection Combining (SC-symbol) using as criterion the estimated SNR, carrier $\mathrm{SC}$ (SC-carrier) using as criterion the maximum of the channel gains of different carriers, and Maximum Ratio Combining (MRC) with and without weighing by the estimated SNR [8]. The HFDVL modem runs in real time on a Linux-based PC, with all the processes related to time and frequency synchronization acquisition and tracking, channel and SNR estimation (actually Signal to Noise+Inteference Ratio) done in parallel, as shown in Figure 2 (b).

\section{Experimental Results}

In three different measurements campaigns, more than 6300 signals were registered and analysed during the first semester of year 2009. The first two were devoted to studies of spatial and polarization diversities. All the combinations were implemented using either OFDM or OFDM-CDM modulations with 4QAM constellation, LDPC1/2 coding or no coding, and block interleaver with length of 1.6, 3.3 and $5.0 \mathrm{sec}$ or no interleaver.

For each of the aforementioned configurations, 40 signals of 1 min each were transmitted in the first campaign and 4 signals of 10 min each were transmitted in the second one. The third campaign was carried out with the same parameters as in the first one but also including band diversity, with a gap between bands of 36, 84, 132 and $324 \mathrm{KHz}$. Due to space limitation, only a small set of the results of the first campaign will be shown here; the main conclusions are very similar for all the signals analysed, and they agree with previous studies carried out as described in the Introduction section (see [3-5].)

Regarding the correlation between the received signals, the conclusions are the following: at location B, correlation between the antennas that are 75 meters apart and with the same polarisation is always less than 0.6 , and the correlation between locations A and B $(40 \mathrm{Km}$ apart $)$ is less than 0.15 . Correlation between different polarisations is in the range 0.3 to 0.4 when measured at the same antenna, and 0.25 to 0.3 when measured between antennas. All these results indicate that the correlation between antennas that are so far apart from each other and the correlation between polarisations at the same antenna are enough to obtain a high gain in system's performance.

Figure 3 shows one of the most interesting results after a massive analysis of the signals, which are in this case, OFDM signals with no coding at transmission. Figure 3 (a) shows the diversity-induced gain in BER with respect to the performance obtained at the different no-diversity schemes at location $\mathrm{B}$, where the combining is performed using the MRC technique weighted by estimated SNR. Abscissas represent the BER obtained for each SISO configuration, whereas ordinates represent the BER obtained after MRC combining. The results represent the average of the BER over the different received OFDM symbols. When points are below the diagonal, represented by the dashed line, a gain is obtained with respect to the SISO case, otherwise we have a loss. The figure shows the antenna configuration which is the predominant component in the combination process, marked as Ant2Pol2. For this antenna configuration, a gain of only half an order of magnitude was obtained. But for the other three configurations, a gain of one order of magnitude or higher was obtained. However, for unstable channels such as HF channels, there is another important parameter to consider, namely link reliability. Reliability must be understood as the percentage of time that it is possible to get a given BER. Figure 3 (b) shows reliability for the same configurations as for Figure 4 (a). Compared to the predominant antenna configuration, reliability of the diversity scheme is nearly $20 \%$ higher (from $40 \%$ of the time to $60 \%$ ), but for the other antenna configurations, the increase is dramatically higher, up to a $55 \%$. In other words, link reliability between $5 \%$ and $40 \%$, obtained for the SISO case, increases to $60 \%$ after MRC combining. It is worth pointing out that the antennas are only 75 meters apart; furthermore, in a SISO system, no a priori knowledge about which antenna configuration will be the predominant component is possible. Regarding the increase in link reliability in the band diversity campaign, the best result was less than $50 \%$ (from $25 \%$ to $75 \%$ ) when the gap between bands was $324 \mathrm{KHz}$.

After all the signals were analysed, we conclude that MRC weighted by the estimated SNR achieves the best performance in most of the cases, although SC-symbol provides also goods results, and in some cases similar performance to MRC. It should be recalled that SC-symbol is the simplest to implement. 


\section{Conclusions}

In practical HF communications, only a limited set of diversity strategies can be implemented. We have studied the performance of multicarrier transmissions with coding in time and frequency together with several combining techniques at the receiver end. Polarisation diversity has low implementation complexity in most cases, and yet provides very good performance when MRC is applied. The performance obtained with polarisation diversity with one antenna is similar to that obtained with spatial diversity resulting from two antennas $40 \mathrm{Km}$ apart. BER gains of more than one order of magnitude are possible although the most interesting results are related to reliability of the communication link, which were shown to improve dramatically. Further, SC-symbol technique, which is the simplest to implement, was shown to achieve performance similar to that of MRC, in some scenarios.

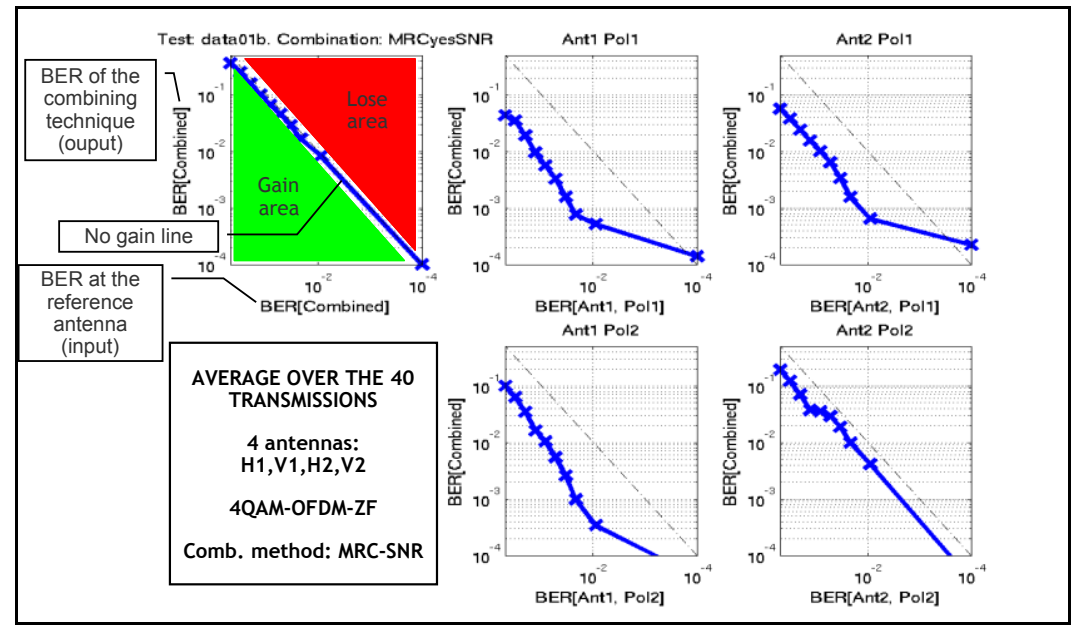

(a)

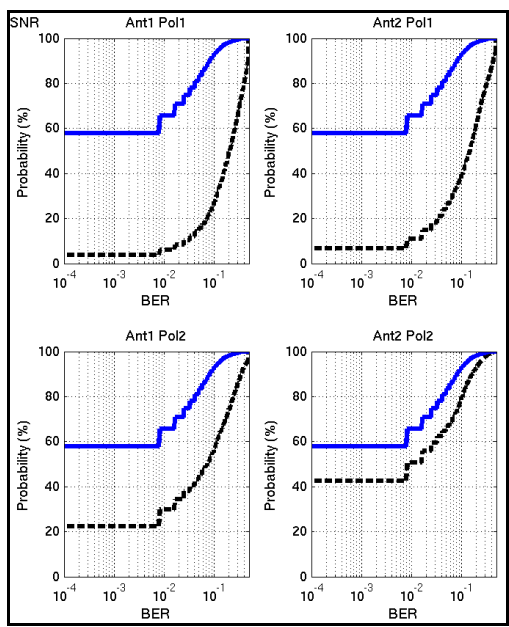

(b)

Figure 3: Analysis for 4QAM-OFDM with no coding and MRC weighted by SNR (a) Average BER performances; (b) Average reliability performances.

\section{Acknowledgments}

This work was partially supported by the Spanish national projects TEC2007-67520-C02, TEC2010-21217-C02 and the mobility grant JC2008-00302. A large part of this work was developed during a stay of the first and fourth authors at the University of Leeds, UK. Authors wish to thank the University of Leeds for its support.

\section{References}

[1] Alamouti, S.M.; "A simple transmit diversity technique for wireless communications" IEEE Journal on Selected Areas in Communications, vol.16, no.8, pp.1451-1458, Oct 1998.

[2] Andreas A. Hutter; Selim Mekrazi; Beza N. Getu; Fanny Platbrood; "Alamouti-based space-frequency condig for OFDM", Springer Wireless Personal Communications, vol 35, no.1-2, pp-173-185, 2005.

[3] Grisdale, G.L.; Morris, J.G.; Palmer, D.S.; , "Fading of long-distance radio signals and a comparison of spaceand polarization-diversity reception in the $6-18 \mathrm{Mc} / \mathrm{s}$ range" Proceedings of the IEE - Part B: Radio and Electronic Engineering, vol.104, no.13, pp.39-51, January 1957.

[4] McLarnon, B.D.; "Performance of simple diversity antenna arrangements in HF data systems," 6th International Conference on Antennas and Propagation vol.2, 4-7 Apr 1989

[5] Jorgenson, M.B.; Johnson, R.W.; Moreland, K.W.; Serinken, N.; Chow, S.; Willink, T.J.; "Polarization diversity for HF data transmission," $7^{\text {th }}$ Int. Conference on HF Radio Systems and Techniques, Nottingham, UK, Jul 1997

[6] Santana, H.; Zazo, S.; Pérez, I.; Raos, I.; Mendieta, E.; López, J. "Validation of a HF Spread Spectrum MultiCarrier Technology through real Link Measurements". European Trans. on Telecommunications (ETT), Vol17, Nov.-Dec. 2006

[7] Pérez-Álvarez, I.; López-Pérez, J.; Zazo; S.; Raos, I.; Pérez-Díaz, B.; Jiménez-Yguacel, E; "Real link of a high data rate OFDM modem: description and performance". 11th International Conference on Ionospheric Radio Systems \& Techniques. IRST 2009, Edinburgh, U.K.; June 2009.

[8] Oestages, C; Clerckx, B.; MIMO wireless communications. From real-world propagation to space-time code design. UK, Elesevier, Academic Press, 2007. 